7. De la Rosette J, Opondo D, Daels F (2012) Categorisation of complications and validation of the clavien score for percutaneous nephrolithotomy. Eur Urol 62:246-255. doi:10.1016/j.eururo.2012.03.055

8. El-Nahas AR, Mansour AM, Ellaithy R et al (2008) Case report: conservative treatment of liver injury during percutaneous nephrolithotomy. J Endourol 22:1649-1652. doi:10.1089/end.2008.0147

9. Ganpule AP, Shah D, Desai MR (2014) Postpercutaneous nephrolithotomy bleeding: aetiology and management. Curr Opin Urol 24:189-194. doi:10.1097/MOU.0000000000000025

10. Gross AJ, Bach T (2009) Preoperative percutaneous stone surgery in patients receiving anticoagulant therapy. J Endourol 23:1563-1565. doi:10.1089/ end.2009.1519

11. Guven S, Istanbulluoglu O, Gul U et al (2011) Successful percutaneous nephrolithotomy in children: multicenter study on current status of its use, efficacy and complications using clavien classification. J Urol 185:1419-1424. doi:10.1016/j.juro.2010.11.055

12. Hopper KD, Sherman JL, Luethke JM (1987) The retrorenal colon in the supine and prone patient. Radiology 162:443-446. doi:10.1148/radiology.162.2.3797658

13. Labate G, Modi P, Timoney A et al (2011) The percutaneous nephrolithotomy global study: classification of complications. J Endourol 25:1275-1280. doi:10.1089/end.2011.0067

14. Lojanapiwat B, Prasopsuk S (2006) Upper-pole access for percutaneous nephrolithotomy: comparison of supracostal and infracostal approaches. J Endourol 20:491-494. doi:10.1089/ end.2006.20.491

15. Michel MS, Trojan L, Rassweiler JJ (2007) Complications in percutaneous nephrolithotomy. Eur Urol 51:899-906. doi:10.1016/j.eururo.2006.10.020

16. Munver R, Delvecchio FC, Newman GE et al (2001) Critical analysis of supracostal access for percutaneous renal surgery. J Urol 166:1242-1246

17. Negrete-Pulido O, Gutierrez-Aceves J (2005) Management of infectious complications in percutaneous nephrolithotomy. J Endourol 23:1757-1762. doi:10.1089/end.2009.1547

18. Patel SR, Haleblian GE, Pareek G (2010) Percutaneous nephrolithotomy can be safely performed in the high-risk patient. Urology 75:51-55. doi:10.1016/j.urology.2009.06.064

19. Radecka E, Brehmer M, Holmgren K et al (2003) Complications associated with percutaneous nephrolithotripsy: supra- versus subcostal access. A retrospective study. Acta Radiol 44:447-451

20. Rassweiler JJ, Müller M, Fangerau M et al (2012) iPad-assisted percutaneous access to the kidney using marker-based navigation: initial clinical experience. Eur Urol 61:628-631. doi:10.1016/j.eururo.2011.12.024

21. Rastinehad AR, Andonian S, Smith AD et al (2009) Management of hemorrhagic complications associated with percutaneous nephrolithotomy. J Endourol 23:1763-1767. doi:10.1089/end.2009.1548

22. Ritter M, Rassweiler MC, Häcker A et al (2013) Laser-guided percutaneous kidney access with the UroDyna-CT: first experience of three-dimensional puncture planning with an ex vivo model. World J Urol 31:1147-1151. doi:10.1007/s00345-0120847-8

23. Ritter M, Krombach P, Michel MS (2011) Percutaneous stone removal. Eur Urol (Suppl) 10(5):433439. doi:10.1016/j.eursop.2011.07.005
24. Shah HN, Hegde S, Shah JN et al (2006) A prospective, randomized trial evaluating the safety and efficacy of fibrin sealant in tubeless percutaneous nephrolithotomy. J Urol 176:2488-2492. doi:10.1016/j.juro.2006.07.148

25. Shah HN, Hegde SS, Mahajan AP et al (2007) Splenic injury: rare complication of percutaneous nephrolithotomy: report of two cases with review of literature. J Endourol 21:919-922. doi:10.1089/ end.2006.0451

26. Singh I, Saran RN, Jain M (2008) Does sealing of the tract with absorbable gelatin (Spongostan) facilitate tubeless PCNL? A prospective study. J Endourol 22:2485-2493. doi:10.1089/end.2008.0321

27. Traxer $O$ (2009) Management of injury to the bowel during percutaneous stone removal. J Endourol 23:1777-1780. doi:10.1089/end.2009.1553

28. Türk C, Knoll T, Petrik A et al (2013) Guidelines on urolithiasis. In: European Association of Urology. Drukkerij Gelderland, Arnhem

29. Wezel F, Mamoulakis C, Rioja J et al (2009) Two contemporary series of percutaneous tractdilatation for percutaneous nephrolithotomy. J Endourol 23:1655-1661

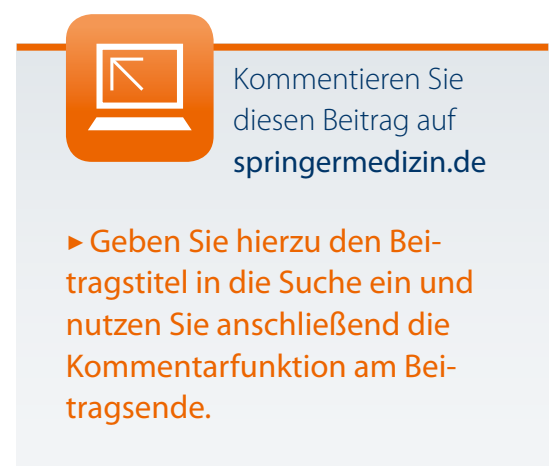

Urologe 2014 · 53:1016

DOI 10.1007/s00120-014-3579-0

Online publiziert: 5. Juli 2014

๑) Springer-Verlag Berlin Heidelberg 2014

K.A. Probst ${ }^{1} \cdot$ K. Böhm ${ }^{2}$

H. Borgmann ${ }^{3} \cdot$ B. Beyer ${ }^{2}$

${ }^{1}$ Klinik für Urologie und Kinderurologie,

Universitätsklinikum des

Saarlandes, Homburg/Saar

2 Universitätsklinikum Hamburg-Eppendorf,

Martini-Klinik, Hamburg

${ }^{3}$ Klinik für Urologie und Kinderurologie,

Universitätsklinikum Frankfurt,

Frankfurt am Main

\section{Erratum zu: YouTube \& Co. Welche Bedeutung haben Videoportale in der modernen Medizin?}

In der HTML-Version zu diesem Beitrag fehlt der englische Beitragstitel. Dieser lautet „YouTube \& Co. Impact of online video portals on contemporary medicine“. Die Redaktion bittet, die korrekte Angabe zu beachten und den Fehler zu entschuldigen.

Die Redaktion

\section{Korrespondenzadresse}

\section{K.A. Probst}

Klinik für Urologie und Kinderurologie, Universitätsklinikum des Saarlandes, Kirrberger Straße, 66424 Homburg/Saar kai.probst@uks.eu

Die Online-Version des Originalartikels können Sie einsehen unter http://dx.doi. org/10.1007/s00120-014-3494-4 